\title{
Propuesta Didáctica Constructivista para la Adquisición de Aprendizajes Significativos de Conceptos en Física de Fluidos
}

\author{
Alicia A. Bravo (1), Glenda P. Ramírez (2); Claudio A. Faúndez ${ }^{(2)}$ y Hernán F. Astudillo(2). \\ (1) Lycée Charles de Gaulle, Concepción, Chile (e-mail: aliciabravo@udec.cl). \\ (2) Depto. De Física, Facultad de Ciencias Físicas y Matemáticas, Universidad de Concepción, \\ Barrio Universitario s/n, Casilla 160-C, Concepción, Chile (e-mail: glendaramirez@udec.cl, \\ claudiofaundez@udec.cl, hastudil@udec.cl).
}

Recibido Ago. 27, 2015; Aceptado Nov. 3, 2015; Versión final Nov. 23, 2015, Publicado Abr. 2016

\begin{abstract}
Resumen
Este trabajo presenta una propuesta didáctica constructivista, basada en la realización de laboratorios experimentales para la unidad de física de fluidos, que fue diseñada por estudiantes de pedagogía en física de la Universidad de Concepción, Chile. La metodología consta de tres etapas: i) teórico- conceptual; ii) experimental y obtención de resultados y iii) análisis de la información y resultados. La propuesta fue implementada, ejecutada y probada en cursos de nivel secundario de un colegio local. Según el factor de Hake, los resultados muestran que los estudiantes obtuvieron una alta ganancia conceptual de aprendizajes observada en el incremento de las calificaciones. Más aún, se observó un cambio en la dinámica de trabajo de los estudiantes, lo que fue verificado por el incremento de la afinidad y la transferencia de aprendizajes en el grupo. La formulación de propuestas didácticas constructivista bajo la metodología experimental muestra ser relevante en el proceso de enseñanza-aprendizaje para la formación universitaria de futuros docentes de ciencias.
\end{abstract}

Palabras clave: física de fluidos, constructivismo, aprendizaje significativo, redes de afinidad, factor de Hake

\section{Didactic Constructivist Proposal to Acquire Significant Learning of Concepts in Physics of Fluid}

\begin{abstract}
This paper presents a constructivist didactic proposal, based on the performance of experimental laboratories of fluid physics unit, designed by students of bachelor of education in natural and physical sciences at the University of Concepción, Chile. The methodology consists of three steps: i) theoretical and conceptual; ii) experimental and data gathering; and iii) data analysis and results. The proposal was implemented, executed and tested in high school courses at a local college. Hake factor indicates that students had high conceptual learning gain, observed in the increase in average scores. Furthermore, a change was observed in the workflow of students, verified by the increased affinity in the group, as well as by the appearance of a learning transfer network. The formulation of didactic constructivist proposals under experimental methodology proves to be relevant in the teaching-learning process of future science teachers.
\end{abstract}

Keywords: fluid physics, constructivist, meaningful learning, networking affinity, Hake factor 


\section{INTRODUCCIÓN}

Las actividades experimentales adquieren gran importancia en el ámbito educacional, ya que son consideradas como mecanismos de motivación, promueven el trabajo en equipo y favorecen la comprensión del conocimiento y razonamiento científico (Hodson, 1994; Barberá y Valdés, 1996; Mordeglia y Mengascini, 2014). Es más, promueven el aprendizaje de las ciencias, ya que consideran un entorno adecuado para el aprendizaje a través de la indagación, resaltando la importancia de integrar lo práctico con lo teórico, haciendo del proceso de enseñanza-aprendizaje una actividad activa y eficaz (Bolaños, 2012; Crujeiras y Jiménez, 2015).

En Chile el Ministerio de Educación (MINEDUC) plantea que para motivar a los estudiantes en el aprendizaje de la ciencia, desarrollar el pensamiento científico y las habilidades asociadas, es necesario exponer a los alumnos a gran cantidad de actividades experimentales. Además, entrega ciertas orientaciones didácticas, propias de la enseñanza de las ciencias, que el profesorado puede poner en práctica para el desarrollo integrado de los contenidos, habilidades y actitudes, con especial énfasis en el aprendizaje de las habilidades de investigación científica y en la indagación (curriculum en línea). Es indispensable que el estudiante tenga experiencias directas con los fenómenos que se están estudiando, asociando la teoría de la clase con lo práctico de las actividades experimentales, ya que la experiencia es la clave para la construcción del conocimiento (Bolaños, 2012).

La enseñanza de las ciencias muchas veces presenta una cierta dificultad en la práctica, puesto que se integran aspectos conceptuales, procedimentales y epistemológicos (Flores et al., 2009). Debido a esto, y considerando la importancia de la ciencia en la vida cotidiana, que el grupo de expertos del área de Ciencias PISA (programe for International Student Assessment, de la OCDE) define el término Alfabetización Científica como "la capacidad de usar el conocimiento científico para identificar preguntas y para sacar conclusiones basadas en las pruebas, con el fin de entender y ayudar a tomar decisiones sobre el mundo natural y los cambios realizados en él a través de la actividad humana" (González Weil et al., 2009). En este sentido, es que la didáctica aplicada a las ciencias cobra relevancia en la entrega de los contenidos y asegurará un aprendizaje significativo en la medida en que los estudiantes se identifiquen con las actividades que desarrollen, resultando favorable para ellos el uso de prácticas experimentales por encima de las guías preestablecidas (Carrasco et al., 2006). De aquí, es que el trabajo práctico en el laboratorio juega un importante rol en el proceso enseñanza-aprendizaje, debido principalmente al desarrollo de diversas habilidades como las descritas por algunos autores recientemente (Ver Tabla 1). Además, el estudiante toma un rol principal y activo, donde se le conduce a hacer uso de sus conocimientos previos y se requiere que trabaje a partir de su experiencia y de los acuerdos a los que puedan llegar en el trabajo grupal, para asi responder a los objetivos de aprendizaje propuestos por el docente (Avendaño et al., 2012; López y Tamayo, 2012).

Tabla 1: Habilidades del trabajo práctico

\begin{tabular}{|l|l|}
\hline Autores & Habilidades \\
\hline Flores et al. (2009) & $\begin{array}{l}\text { Discutir, razonar y comparar lo que se ha hecho en el trabajo práctico, teniendo así } \\
\text { la oportunidad de vivir un proceso real de resolución de problemas. }\end{array}$ \\
\hline Avendaño et al. (2012) & $\begin{array}{l}\text { Expresión, argumentación, trabajo en equipo análisis y reflexión sobre cada una de } \\
\text { las fases de la resolución de un problema. }\end{array}$ \\
\hline López y Tamayo (2012) & $\begin{array}{l}\text { Trabajo en equipo, el establecimiento de relaciones significativas entre las } \\
\text { actividades prácticas propuestas y la vida cotidiana de los estudiantes y las } \\
\text { relaciones entre el campo específico de la actividad práctica (biología, química, física) } \\
\text { con otros campos del conocimiento. }\end{array}$ \\
\hline
\end{tabular}

Respecto al logro de objetivos en el trabajo de laboratorio es necesario establecer la relación entre el constructivismo y las actividades experimentales y como se favorece el aprendizaje entre pares (Flores et al., 2009; Jaime y Escudero., 2011; Faúndez et al., 2015). En la Tabla 2 se muestra las concepciones de las prácticas de laboratorio desde enfoques instruccional y constructivista.

En la actualidad, las preocupaciones de profesores e investigadores está centrada en el desarrollo de metodologías y estrategias adecuadas para un curso, con énfasis en el desarrollo de la actividad experimental en el laboratorio (Barolli et al., 2010). Sin embargo, uno de los principales problemas que enfrentan los docentes de física cuando imparten clases en el aula secundaria, es la realización de actividades experimentales. En el caso de Chile, existen muy pocos colegios que cuentan con los recursos necesarios para realizar actividades experimentales asociadas a los contenidos mínimos obligatorios, exigidos por el MINEDUC. Cofré et al. (2010) menciona que en Chile existen programas de apoyo como ECBI (Enseñanza de las Ciencias Basada en Indagación) y MECIBA (Mejoramiento de las Ciencias en la Enseñanza Básica) que están colaborando de manera importante al mejoramiento de la enseñanza de las ciencias en nuestro 
país, pero su cobertura es limitada, tanto en número de escuelas dentro de los programas como al nivel educativo en el que están enfocado.

Tabla 2: Concepción de las prácticas experimentales (López y Tamayo, 2012).

\begin{tabular}{|l|l|}
\hline \multicolumn{1}{|c|}{ Perspectiva Instruccional } & \multicolumn{1}{|c|}{ Perspectiva constructivista } \\
\hline $\begin{array}{l}\text { Confirman algo ya visto en una lección de tipo } \\
\text { expositivo. }\end{array}$ & $\begin{array}{l}\text { El profesor debe actuar como guía, facilitando el proceso de } \\
\text { aprendizaje. }\end{array}$ \\
\hline $\begin{array}{l}\text { Las prácticas son el único criterio de validez del } \\
\text { conocimiento científico y la prueba definitiva de las } \\
\text { hipótesis y teorías. }\end{array}$ & $\begin{array}{l}\text { La experiencia tiene un rol importante, pero por sí sola no } \\
\text { puede rechazar o verificar las hipótesis. Entre la teoría y el } \\
\text { experimento no se establecen jerarquías. }\end{array}$ \\
\hline $\begin{array}{l}\text { Exigir que los estudiantes sigan una receta para llegar } \\
\text { a una conclusión predeterminada. }\end{array}$ & $\begin{array}{l}\text { El profesor debe informarse sobre las ideas previas, } \\
\text { habilidades y dificultades que tienen los estudiantes. }\end{array}$ \\
\hline $\begin{array}{l}\text { Percibir el laboratorio como lugar donde se hacen } \\
\text { cosas, pero no se comunica a los estudiantes el } \\
\text { significado de lo que se hace. }\end{array}$ & $\begin{array}{l}\text { El profesor debe centrar su atención en aspectos sociales del } \\
\text { aprendizaje (entender la ciencia como una estructura social). }\end{array}$ \\
\hline $\begin{array}{l}\text { Proceder ciegamente a tomar apuntes o a manipular } \\
\text { aparatos sin tener un propósito claro. }\end{array}$ & Elección de experiencias científicas apropiadas para el aula. \\
\hline
\end{tabular}

En física, la mecánica de fluidos, estudia el comportamiento de líquidos y gases en estado de reposo y movimiento. A nivel secundario, la física de fluidos, parece ser más complicada de lo que es, ya que su enseñanza resulta ser abstracta si no se combina con una metodología experimental. Debido a que estos contenidos presentan variados tópicos que pueden ser explicados con la ayuda de laboratorios, existen algunos trabajos que han sido presentados en la literatura, que muestran la aplicación de algunas actividades experimentales. La Tabla 3 muestra una breve descripción de trabajos sobre la enseñanza de la física de fluidos.

Tabla 3. Algunos trabajos propuestos sobre enseñanza de física de fluidos.

\begin{tabular}{|c|c|c|}
\hline Autores & Tema & Descripción \\
\hline $\begin{array}{l}\text { Barbosa, } \\
2003 .\end{array}$ & $\begin{array}{l}\text { Ecuación de } \\
\text { Bernoulli }\end{array}$ & $\begin{array}{l}\text { Presentan un experimento discrepante sobre el estudio de la Ecuación de Bernoulli, cuyo } \\
\text { objetivo es cautivar el interés del estudiante e insertarlo en el estudio de los fluidos. El } \\
\text { trabajo fue desarrollado con estudiantes de primer semestre de física, donde se } \\
\text { implementó un enfoque constructivista en la observación, conjeturización y análisis de la } \\
\text { fenomenología. }\end{array}$ \\
\hline $\begin{array}{l}\text { Arnau, } \\
2008 .\end{array}$ & $\begin{array}{l}\text { Fuerza } \\
\text { flotación }\end{array}$ & $\begin{array}{l}\text { Se presenta la construcción de un barco de movimiento producido por una hélice aérea, } \\
\text { movida por un motor que se alimenta mediante una placa solar. La experiencia se realizó } \\
\text { con estudiantes secundarios, donde la intención es que los alumnos aprendan, en un } \\
\text { contexto completamente aplicado, algunos elementos básicos de mecánica de fluidos, } \\
\text { hidrostática, mecánica y células fotovoltaicas. }\end{array}$ \\
\hline $\begin{array}{l}\text { Belandria } \\
\text { et al., } \\
2010 \text {. }\end{array}$ & $\begin{array}{l}\text { Mecánica de } \\
\text { Fluidos }\end{array}$ & $\begin{array}{l}\text { Realizan una investigación cuyo objetivo es la planificación, desarrollo y evaluación de un } \\
\text { proyecto didáctico para el trabajo de conceptos sobre mecánica de fluidos. La metodología } \\
\text { utilizada fue de tipo cualitativo y considero un total de } 47 \text { estudiantes de educación inicial, } \\
\text { primaria y secundaria. Se muestra que el aprendizaje resulta más efectivo cuando los } \\
\text { estudiantes confrontan sus ideas con experiencias prácticas. }\end{array}$ \\
\hline $\begin{array}{l}\text { Mosquera, } \\
2011 .\end{array}$ & $\begin{array}{l}\text { Fluidos en el } \\
\text { sistema } \\
\text { circulatorio }\end{array}$ & $\begin{array}{l}\text { Aborda el estudio de los procesos físicos en el transporte de fluidos; a través del sistema } \\
\text { circulatorio. El estudio se realizó con estudiantes de secundaria. Los resultados muestran } \\
\text { que los estudiantes encuentran el significado de lo que aprenden, ya que establecen un } \\
\text { vínculo tangible entre el concepto y el proceso físico que se da en el cuerpo humano. }\end{array}$ \\
\hline $\begin{array}{l}\text { Szigety } \\
\text { et al., } \\
2012 \text {. }\end{array}$ & $\begin{array}{l}\text { Tensión } \\
\text { superficial }\end{array}$ & $\begin{array}{l}\text { Presentan una propuesta basada en una actividad experimental, cuyo objetivo es lograr } \\
\text { que los alumnos comprendan e interpreten el fenómeno de tensión superficial. La } \\
\text { propuesta fue realizada por alumnos de enseñanza secundaria en la clase de física } \\
\text { durante el desarrollo del contenido de mecánica de fluidos. }\end{array}$ \\
\hline $\begin{array}{l}\text { Avendaño } \\
\text { et al., } \\
2012 \text {. }\end{array}$ & \begin{tabular}{|l|} 
Túnel \\
Viento
\end{tabular} & $\begin{array}{l}\text { Presentan el diseño e implementación de cinco módulos de laboratorio, uno de ellos sobre } \\
\text { el tema del túnel de viento. La propuesta fue desarrollada en dos instituciones secundarias } \\
\text { con estudiantes de diferentes grados. El trabajo experimental consta de diseño, } \\
\text { construcción, funcionamiento del dispositivo y análisis de conceptos físicos. }\end{array}$ \\
\hline
\end{tabular}

En el presente trabajo se da a conocer una propuesta didáctica desarrollada para la unidad de fluidos. Dicha propuesta, además de generar conocimiento requerido, potencia las habilidades de los estudiantes universitarios permitiendo motivar la curiosidad de sus futuros alumnos. La propuesta está diseñada por 
estudiantes de la carrera de pedagogía en ciencias naturales y física de la Universidad de Concepción y nace como una motivación para aportar material didáctico y experimental, que como es conocido, en las escuelas secundarias de Chile, los docente en general, no cuentan con guías de trabajo experimental validadas que permitan a los estudiantes construir su propio conocimiento a través de experimentos sencillos (Vera et al., 2013). Este trabajo fue desarrollo en la asignatura de proyecto de física II, para ser aplicada como una actividad curricular enfocada a alumnos de tercero año de la enseñanza media chilena, donde los estudiantes realizaran sesiones donde cada contenido se desarrolla en tres etapas: 1) teórico- conceptual; 2) experimental y 3) análisis de la información y formulación de resultados. En las siguientes secciones se describe el diseño de la propuesta y se muestra un análisis de los resultados y las conclusiones obtenidas.

\section{METODOLOGÍA}

La asignatura de proyecto de física II, que se encuentra dirigida a estudiantes de pedagogía en ciencias naturales y física de la Universidad de Concepción, tiene por objetivo elaborar proyectos de mejoramiento para la enseñanza de las ciencias. En el primer semestre del año 2014, se llevó a cabo la elaboración de propuestas didácticas centradas particularmente en el aprendizaje por indagación, mediante la utilización de laboratorios experimentales. Para desarrollar estas actividades, los estudiantes universitarios consideraron la integración de los conceptos teóricos en su práctica profesional, siguiendo los lineamientos de los contenidos entregados por el MINEDUC para los estudiantes de tercer año de enseñanza media en Chile (ver Tabla 4). Este trabajo fue realizado en grupos, considerando por parte del docente la metodología del aprendizaje de tipo cooperativo (Parra y Flores, 2008; Lazzari, 2014).

Tabla 4: Contenidos tercer año medio

\begin{tabular}{|c|c|c|c|}
\hline Semestre & Unidad & Contenidos & Descripción \\
\hline \multirow[b]{2}{*}{ I } & \multirow[b]{2}{*}{ Mecánica } & 1. Movimiento circular & $\begin{array}{l}\text { a) Descripción del movimiento circular uniforme. } \\
\text { b) Dinámica del movimiento circular uniforme. } \\
\text { c) Nociones sobre el momento angular. }\end{array}$ \\
\hline & & $\begin{array}{l}\text { 2. Conservación de la } \\
\text { energía mecánica }\end{array}$ & $\begin{array}{l}\text { a) La energía mecánica en la caída libre. } \\
\text { b) Estudio de la energía en la montaña rusa. } \\
\text { c) Energía mecánica y roce. } \\
\text { d) Resolución de problemas aprovechando la ley de } \\
\text { conservación de la energía mecánica. }\end{array}$ \\
\hline \multirow[t]{2}{*}{ II } & \multirow[t]{2}{*}{ Fluidos } & 1. Hidrostática & $\begin{array}{l}\text { a) Descripción general de los fluidos. } \\
\text { b) Presión hidrostática. } \\
\text { c) Principio de Arquímedes. } \\
\text { d) La Capilaridad. } \\
\text { e) Tensión Superficial. }\end{array}$ \\
\hline & & 2. Hidrodinámica & $\begin{array}{l}\text { a) Las leyes de Bernoulli. } \\
\text { b) Roce y velocidad límite. } \\
\text { c) Presión sanguínea. } \\
\text { d) Los científicos y sus contribuciones. }\end{array}$ \\
\hline
\end{tabular}

Durante el desarrollo de la asignatura, los estudiantes adquirieron las siguientes competencias asociadas a este diseño educativo: i) construcción de un informe sobre la propuesta de laboratorio que incluya su fundamento teórico y científico, metodología y presupuesto; ii) exposición a sus pares y profesor para una retroalimentación; iii) realización del montaje e implementación de su trabajo, recogiendo las sugerencias aportadas. Mediante la utilización de instrumentos de evaluación, aplicados a grupos de 2 a 3 estudiantes universitarios, se corroboró la adquisición de todas estas competencias. Además, los estudiantes adquirieron conceptos sobre sistemas complejos y herramientas de cálculo, que son de gran utilidad cuando se requiere verificar la eficacia de su propuesta. Como se puede apreciar de la Tabla 4, la unidad de fluidos tiene una extensión correspondiente a un semestre completo, por lo que existe tiempo suficiente para que los estudiantes logren adquirir completa y significativamente los contenidos de la asignatura. Los laboratorios experimentales propuestos por los estudiantes universitarios cubren la segunda unidad de fluidos, donde se realizan diversas actividades prácticas que contemplan contenidos de hidrostática e hidrodinámica que son adquiridos por estudiantes secundarios en clases. La Tabla 5 presenta un resumen sobre los contenidos y actividades que los estudiantes universitarios planificaron y realizaron en su propuesta didáctica. Para cada una de estas actividades experimentales desarrolladas por los estudiantes universitarios, se realizaron laboratorios donde los estudiantes secundarios fueron los principales actores a la hora de aplicar conceptos para obtener conclusiones respecto a su trabajo. Cabe mencionar, que dentro de la línea constructivista, este tipo de actividades favorecen el aprendizaje significativo de los estudiantes a través del aprender haciendo (ver por ejemplo, Faúndez et al., 2015). Además, la implementación de estas actividades teórico-prácticas permiten el desarrollo de los contenidos temáticos, actitudinales y procedimentales (Bolaños, 2012). 
Tabla 5: Contenidos de fluidos realizados con laboratorios experimentales.

\begin{tabular}{|l|l|l|}
\hline \multicolumn{1}{|c|}{ Contenido } & \multicolumn{1}{|c|}{ Actividad experimental } & \multicolumn{1}{c|}{ Objetivos } \\
\hline Principio de Arquímedes & Medición de volúmenes & Comprender el principio de Arquímedes. \\
\hline Capilaridad & Claveles de colores & Comprender como actúa la capilaridad en un fluido. \\
\hline Tensión Superficial & Clip flotante & Verificar la acción de la tensión superficial. \\
\hline Caudal & Remolinos de agua & $\begin{array}{l}\text { Identificar el tipo de relación que existe entre el área } \\
\text { transversal y la velocidad. }\end{array}$ \\
\hline Aplicación de Bernoulli & Aerodinámica de vuelo & $\begin{array}{l}\text { Verificar la influencia de la presión en el funcionamiento de } \\
\text { las alas de los aviones. }\end{array}$ \\
\hline $\begin{array}{l}\text { Roce viscoso y } \\
\text { velocidad límite }\end{array}$ & Esferas en fluidos & $\begin{array}{l}\text { Verificar las características de cada fluido cuando un cuerpo } \\
\text { cae dentro de estos. }\end{array}$ \\
\hline
\end{tabular}

Cada una de las actividades experimentales fueron realizadas en grupos, por los estudiantes secundarios, donde fueron ellos mismos quienes eligieron con quien trabajar según su grado de afinidad. La Tabla 6 muestra la descripción de cada actividad experimental propuesta por los estudiantes universitarios en la asignatura de proyecto de física II, la cual fue aplicada posteriormente en la enseñanza de fluidos a nivel secundario. Cada una de estas actividades experimentales tiene una duración de 2 horas.

Tabla 6: Descripción de actividades experimentales

\begin{tabular}{|c|c|c|}
\hline Actividad Experimental & Descripción de actividad & Materiales \\
\hline $\begin{array}{l}\text { Medición } \\
\text { de volúmenes }\end{array}$ & $\begin{array}{l}\text { Los estudiantes trabajaron con probetas con distintos } \\
\text { niveles de agua, donde deben anotar el volumen inicial para } \\
\text { luego colocar distintos cuerpos geométricos con volumen } \\
\text { conocido dentro de la probeta y anotar el volumen final (Fig. } \\
\text { 1). Luego comprobaron teóricamente el volumen de los } \\
\text { cuerpos conocidos con el experimental y así verificar el } \\
\text { principio de Arquímedes. Repitieron la acción con cuerpos } \\
\text { de volumen desconocido. Como segunda actividad } \\
\text { calcularon la densidad de los cuerpos y corroborar su valor } \\
\text { con la teoría. }\end{array}$ & $\begin{array}{l}2 \text { cuerpos sólidos con volumen } \\
\text { conocido y } 2 \text { cuerpos sólidos } \\
\text { con volumen desconocido, } \\
\text { probetas, balanza, vernier. }\end{array}$ \\
\hline $\begin{array}{l}\text { Claveles } \\
\text { de colores }\end{array}$ & $\begin{array}{l}\text { Los estudiantes trabajaron con } 2 \text { tubos de ensayos con } \\
\text { agua y colorante, para luego introducir los claveles blancos } \\
\text { y dejarlos ahí por el resto del tiempo. Al final la actividad los } \\
\text { alumnos observaron que los claveles están teñidos del color } \\
\text { del colorante y verificaron el concepto de capilaridad (Fig.2). }\end{array}$ & $\begin{array}{l}2 \text { claveles, tubo de ensayo y } \\
\text { soporte, colorante vegetal, } \\
\text { cortante. }\end{array}$ \\
\hline Clip flotante & $\begin{array}{l}\text { Los estudiantes trabajaron con una cubeta de agua, donde } \\
\text { en primera instancia colocan un clip en la superficie del agua } \\
\text { el cual cae al fondo rápidamente. En una segunda instancia } \\
\text { colocaron un trozo de papel higiénico sobre la superficie del } \\
\text { agua e introdujeron suavemente el clip; luego sacaron con } \\
\text { mucho cuidado el papel y el clip queda flotando. Analizaron } \\
\text { la situación para corroborar el concepto de tensión } \\
\text { superficial. }\end{array}$ & $\begin{array}{l}\text { Un clip, un recipiente con agua } \\
\text { (puede ser un vaso), un trozo } \\
\text { de papel higiénico muy fino o } \\
\text { un pañuelo desechable. }\end{array}$ \\
\hline $\begin{array}{l}\text { Remolinos } \\
\text { de agua }\end{array}$ & $\begin{array}{l}\text { Los estudiantes realizaron un recipiente con distintas área } \\
\text { (una mayor y una menor) y una compuerta movible, donde } \\
\text { en cada una colocaron molinos de palos de helados. Luego } \\
\text { lo llenaron de agua y al abrir la compuerta los molinos } \\
\text { comenzaron a girar. Los estudiantes verificaron el } \\
\text { cumplimiento de la ecuación de continuidad. }\end{array}$ & $\begin{array}{l}\text { Dos cajas de tetrapak de un } \\
\text { litro (vacías y limpias), scotch o } \\
\text { huincha aisladora, palos de } \\
\text { helado, cartón grueso, un lápiz } \\
\text { marcador permanente, dos } \\
\text { trozos rectangulares de } \\
\text { plumavit grueso de } 10 \times 5 \mathrm{~cm} \text {, } \\
\text { tijeras, dos alambres gruesos o } \\
\text { dos palos para brochetas. }\end{array}$ \\
\hline Aerodinámica de vuelo & $\begin{array}{l}\text { Los estudiantes realizaron una maqueta de las alas de un } \\
\text { avión con hojas de papel y cartón, luego las colgaron de un } \\
\text { hilo fijo para colgarlas sobre un dinamómetro. Finalmente } \\
\text { las colocaron frente a un ventilador y analizaron la situación } \\
\text { respecto a presiones y velocidades. }\end{array}$ & $\begin{array}{l}\text { Papel, cartón, pegamento, un } \\
\text { dinamómetro, hilo, un } \\
\text { ventilador. }\end{array}$ \\
\hline $\begin{array}{l}\text { Esferas } \\
\text { en fluidos }\end{array}$ & $\begin{array}{l}\text { Los estudiantes tienen } 4 \text { probetas con distintos fluidos, } \\
\text { donde dejaron caer pequeñas esferas de aluminio para } \\
\text { estudiar y analizar los conceptos de viscosidad, velocidad } \\
\text { límite y fuerza de roce viscoso (Fig. } 3 \text { ). }\end{array}$ & $\begin{array}{l}\text { Tres probetas transparentes, } \\
\text { agua, aceite, glicerina, tres } \\
\text { bolitas de acero de distinto } \\
\text { tamaño. }\end{array}$ \\
\hline
\end{tabular}




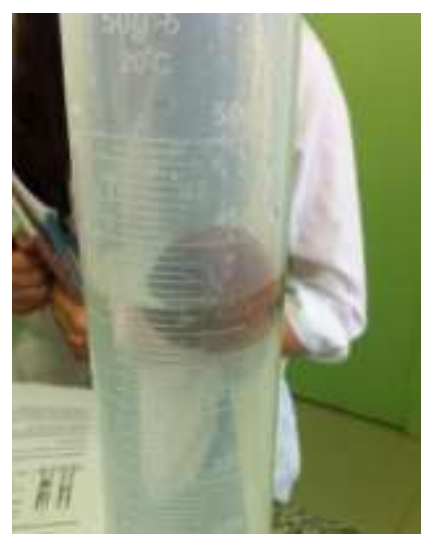

Fig.1: Cálculo de volumen con cuerpo sólido conocido.

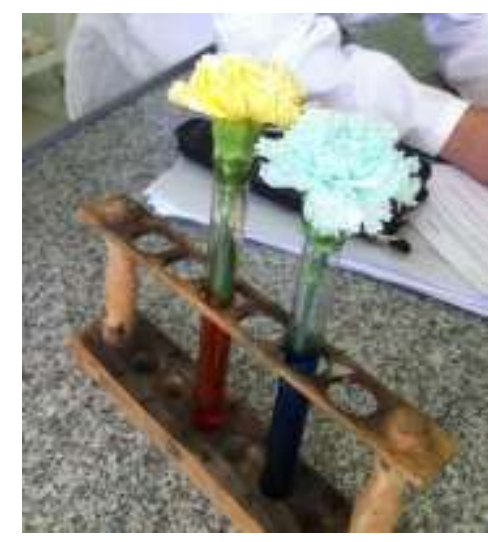

Fig. 2: Claveles teñidos para comprobar la capilaridad.

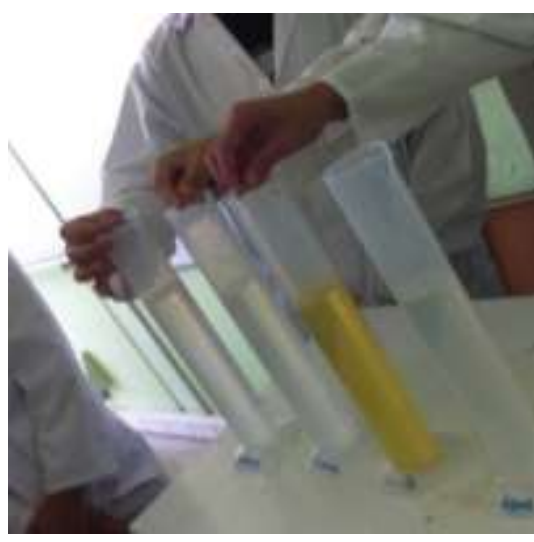

Fig. 3: Estudiantes dejando caer esferas metálicas en probetas que contienen diferentes líquidos.

Dado que las actividades de laboratorio son consideradas cognitivamente complejas, debido a lo que implica la solución de un situación problemática experimental (Escudero y Jaime, 2011), los estudiantes de la carrera de pedagogía decidieron realizar guías de apoyo para los estudiantes secundarios y les solicitaron realizar un informe grupal, bajo un formato flexible que incluía formulación de hipótesis, planificación de la experiencia, análisis de datos y elaboración y presentación de conclusiones.

\section{RESULTADOS y DISCUSIÓN}

Como una forma de validar la propuesta didáctica presentada por los estudiantes universitarios, se implementó este conjunto de actividades experimentales, donde los laboratorios fueron aplicados en un colegio de la octava región de Chile. En la Tabla 7 se describen las características del grupo.

Tabla 7: Descripción del grupo de aplicación de los laboratorios experimentales.

\begin{tabular}{|l|l|l|}
\hline & \multicolumn{1}{|c|}{ Año 2014 } & \multicolumn{1}{c|}{ Año 2015 } \\
\hline Población & 16 alumnos & 20 alumnos \\
\hline Nivel & tercer año medio & tercer año medio \\
\hline Institución & Colegio particular & Colegio particular \\
\hline Región & Del Biobío & Del Biobío \\
\hline Implementación & Física, plan electivo & Física, plan electivo \\
\hline Duración & 1 trimestre & 1 trimestre \\
\hline Aplicación & $1^{\text {er }}$ trimestre & $1^{\text {er }}$ trimestre \\
\hline
\end{tabular}

Tal como indica la Tabla 7 los laboratorios experimentales se aplicaron durante el primer trimestre del año escolar 2014 y 2015, de acuerdo a los contenidos descritos en la Tabla 5. El ambiente de aprendizaje se conformó en el aula, bajo la guía de uno de los alumnos universitarios proponentes de este trabajo. Los contenidos teóricos fueron entregados en el aula y las actividades experimentales que se muestran en la Tabla 6, fueron realizadas en el laboratorio de ciencias de la institución. La propuesta didáctica considera un grupo control donde sólo se entregan contenidos teóricos, esto basado en la enseñanza tradicional (ver por ejemplo Faúndez et al., 2014).

Para verificar el aprendizaje de los contenidos, los estudiantes fueron evaluados según los criterios de evaluación planteados por el MINEDUC, con un pre test al inicio de la unidad y un post test al finalizar la unidad. Además, se evaluó su trabajo en el laboratorio con informes escritos. Cabe mencionar que estas evaluaciones se aplicaron a los cursos donde se implementaron las actividades experimentales, al igual que al grupo control. Para verificar la eficacia de la adquisición de aprendizaje significativo, mediante la implementación de los laboratorios experimentales, se utiliza el factor "g" de Hake (1998), que es un indicador estadístico de cuanto han aprendido los estudiantes dentro del contexto de una metodología didáctica en particular. La Tabla 8 muestra como considerar este factor. 
Tabla 8: Criterios a considerar respecto a la ganancia (Hake, 1998).

\begin{tabular}{|c|c|}
\hline Criterio & Índice \\
\hline $\mathrm{g}<0,3$ & Bajo \\
\hline $0,7>\mathrm{g} \geq 0,3$ & Medio \\
\hline $\mathrm{g} \geq 0,7$ & Alto \\
\hline
\end{tabular}

La Tabla 9 muestra el análisis de las notas del pre y post test logradas por los estudiantes secundarios según el factor de Hake. Esta Tabla muestra que los estudiantes que finalizaron sus aprendizajes con experiencias de laboratorio obtuvieron una alta ganancia conceptual del aprendizaje adquirido a diferencia de los que no tuvieron actividades prácticas. Cabe destacar que en el establecimiento, el año 2015, sólo existe un curso por nivel, por lo que no hay grupo control.

Tabla 9: Ganancia conceptual según factor de Hake.

\begin{tabular}{|l|l|c|}
\hline & Curso & Ganancia \\
\hline Con actividades experimentales & $3^{\circ}$ medio año 2014 & 0,58 \\
\cline { 2 - 3 } & $3^{\circ}$ medio año 2015 & 0,87 \\
\hline Sin actividades experimentales & $3^{\circ}$ medio año 2014 & 0,32 \\
\hline
\end{tabular}

Debido a que las experiencias de laboratorio fueron realizadas en grupos (3 a 4 alumnos), los estudiantes secundarios eligieron con quien trabajar según su afinidad. Esto con el fin de generar un colectivo en donde cada estudiante logró apropiarse de los aprendizajes esperados. Por ello, una forma de analizar los resultados de esta experiencia fue examinar las redes de afinidad previa y posterior al trabajo experimental y las redes de aprendizaje significativo en el curso del año 2015 donde se realizaron las actividades experimentales.
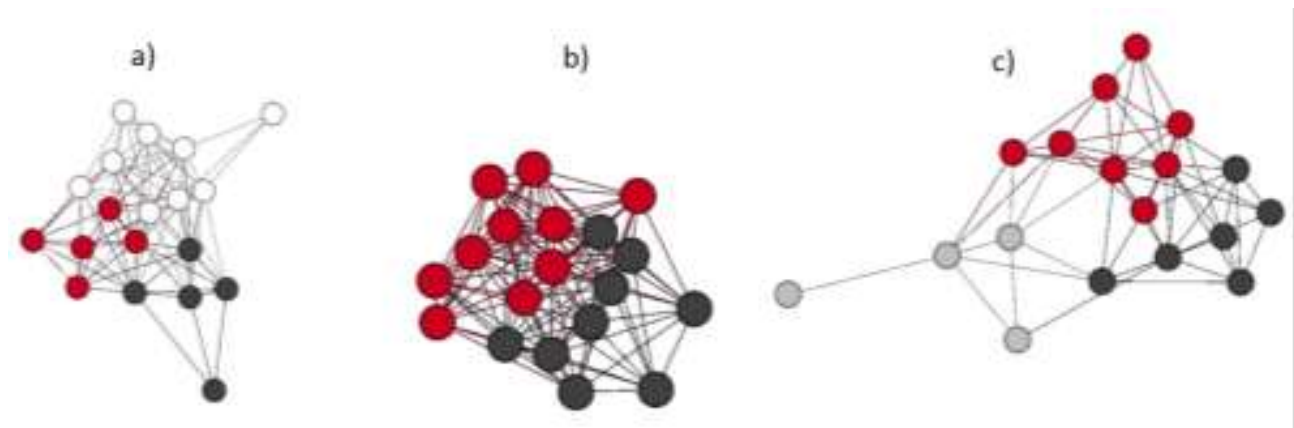

Fig. 4: a) red de afinidad previa; b) red de afinidad posterior y c) red de colaboración.

De la figura 4, se concluye por inspección de los grafos a) y b) que la diversidad observada se reduce de tres grupos a dos con un notorio aumento del número de enlaces, significando ello que, al menos momentáneamente, los lazos de afinidad del grupo se intensificaron. Durante el proceso del intercambio de aprendizajes el grafo c) de la red de colaboración muestra el mismo número de poblaciones por lo que se puede decir que tiene una estructura similar a la red de afinidad previa, mas, la red de colaboración tiene un número menor de enlaces. Esto se interpreta fácilmente por medio del orden observado, donde la información acerca de los aprendizajes tiene menos caminos disponibles que el intercambio social. Una descripción más detalladas sobre redes de afinidad puede encontrarse en el trabajo de Astudillo et al. (2015).

Además, teniendo en cuenta que el sistema educacional chileno considera las calificaciones de los estudiantes como factor de aprendizaje, se ha analizado las calificaciones obtenidas por los estudiantes del grupo experimental y del grupo control obteniéndose los resultados mostrados en la Tabla 10. Notar que la escala de notas va de 1,1 a 7,0 según lo establecido por el MINEDUC. A pesar de que en las tres situaciones descritas los estudiantes obtienen un aumento en sus calificaciones en el post test, los estudiantes que realizaron actividades experimentales muestran un aumento mucho más significativo que el grupo control, lo que además se confirma con el factor de Hake descrito anteriormente.

Tabla 10: Calificaciones obtenidas por los estudiantes con y sin laboratorios experimentales.

\begin{tabular}{|l|c|c|c|c|}
\hline & Año & Promedio pre test & Promedio post test & Informe laboratorio \\
\hline \multirow{2}{*}{$\begin{array}{l}\text { Con actividades } \\
\text { experimentales }\end{array}$} & 2014 & 5,7 & 6,5 & 6,8 \\
\cline { 2 - 5 } & 2015 & 5,9 & 6,9 & 7,0 \\
\hline Sin actividades experimentales & 2015 & 5,5 & 6,0 & Sin informe \\
\hline
\end{tabular}

El análisis de resultados, indica que se cumple con los aprendizajes esperados y se concluye que los estudiantes secundarios lograron adquirir todos los contenidos mínimos obligatorios exigidos por el 
MINEDUC. De esta forma es posible cerrar el proceso de enseñanza-aprendizaje con contenidos pertinentes seleccionados por los estudiantes universitarios, generando un grato ambiente de aprendizaje en el aula y potenciando el trabajo colectivo.

\section{CONCLUSIONES}

Se presenta el desarrollo, implementación y ejecución de una propuesta didáctica constructivista, para la enseñanza de conceptos de física de fluidos mediante actividades experimentales, en contenidos como principio de Arquímedes, capilaridad, tensión superficial, ecuación de continuidad, principio de Bernoulli, roce viscoso y velocidad limite. Dicha propuesta fue diseñada por estudiantes universitarios durante el curso de la asignatura proyecto de física II, incluida en la malla curricular de la carrera de pedagogía en ciencias naturales y física de la Universidad de Concepción. De lo anterior, se pueden extraer las siguientes conclusiones:

i) Se presenta evidencia comprobable, de que la creación de propuestas didácticas contribuye a la motivación e interés del estudiante universitario en la asignatura, fomenta el trabajo colaborativo y ayuda a adquirir competencias como diseño e implementación de laboratorios, conceptos de física de fluidos y de redes complejas y herramientas de cálculo.

ii) Se comprobó que esta propuesta didáctica ayudo a los estudiantes secundarios para la obtención de una alta ganancia conceptual de aprendizajes adquiridos y generó una estructura favorable para el aprendizaje en el aula.

iii) La vinculación de la educación superior y la educación secundaria junto con la investigación y la práctica muestran ser de gran importancia en la formación inicial del futuro docente.

iv) Este tipo de actividades debe considerarse fundamental en la formación universitaria de los estudiantes de carreras de pedagogía en ciencias, ya que el desarrollo de este tipo de propuestas didácticas, que involucran trabajo colaborativo, resulta ser relevante para el aprendizaje significativo.

\section{AGRADECIMIENTOS}

Los autores agradecen a la Vicerrectoría de Investigación a través del proyecto $n^{\circ} 215.011 .060-1.0$, al Departamento de Física y a la Dirección de Docencia de la Universidad de Concepción por el apoyo en la construcción de este trabajo.

\section{REFERENCIAS}

Astudillo, H. F., Jara, C., Navarrete, M., Faúndez, C.A., Evidencia de Auto Aprendizaje como Manifestación de un Comportamiento Colectivo Espontáneo en el Aula, Formación Universitaria, 8(2), 43-50, (2015)

Avendaño, R., Lancheros, W., Castiblanco, O. y Arcos, F., La enseñanza de la física a través de módulos experimentales, Góndola, 7(1), 32-49, (2012)

Barberá, O., y Valdés, P., El trabajo práctico en la enseñanza de las ciencias: una revisión. Enseñanza de las Ciencias, Vol. 14, 365-379, (1996)

Barbosa, L., Un movimiento Discrepante en el estudio de una ley de la física de fluidos: La ecuación de Bernoulli, Revista Colombiana de Física, 35(1), 95-98, (2003)

Barolli, E., Laburú, C. y Guridi, V., Laboratorio didáctico de ciencias: caminos de investigación. Revista Electrónica de Enseñanza de las Ciencias, 9(1), 88-110, (2010)

Belandria, R., Bonilla, J., Carrillo, T. y Escalona, J., Educación Inclusiva. Alternativa didáctica: la educación inclusiva "una experiencia con mecánica de fluidos". Congreso lberoamericano de Educación. METAS 2021. Argentina, Buenos Aires, 13-15 Septiembre, (2010)

Bolaños, F., Laboratorio de física mecánica de fluidos como herramienta pedagógica. Ingeniería solidaria, 8(14), 26-33, (2012)

Cofré, H., Camacho, J., Galaz, A., Jiménez, J., Santibáñez, D., Vergara, C., La educación científica en Chile: debilidades de la enseñanza y futuros desafíos de la educación de profesores de ciencia. Estudios pedagógicos XXXVI, 2, 279-293, (2010) 
Crujeiras, B y Jiménez, M., Desafíos planteados por las actividades abiertas de indagación en el laboratorio: articulación de conocimientos teóricos y prácticos en las prácticas científicas, Enseñanza de las ciencias: revista de investigación y experiencias didácticas, 33(1), 63-84, (2015)

Curriculum en línea, orientaciones didácticas en ciencias naturales (en la web: http://www.curriculumenlineamineduc.cl/605/w3-article-20951.html, acceso el 26 de julio de 2015), Ministerio de educación, Chile.

Faúndez, C. A., Bravo, A. A., Melo, A. D., y Astudillo, H. F., Laboratorio Virtual para la Unidad Tierra y Universo como Parte de la Formación Universitaria de Docentes de Ciencias. Formación universitaria, 7(3), 33-40, (2014)

Faúndez, C. A., Rojas, Y. G., Pinto, A. A., y Astudillo, H. F., Taller de Física Cuántica: un Método para Introducir Conceptos Fundamentales en una Actividad Extracurricular. Formación universitaria, 8(2), 53-62, (2015)

Flores, J., Caballero, M. y Moreira, M., El laboratorio en la enseñanza de las ciencias: Una visión integral en este complejo ambiente de aprendizaje. Revista de investigación., 33 (68), 75-111, (2009)

González-Weil, C.; Martínez, M.; Martínez, C.; Cuevas, K.; Muñoz, L., La educación como apoyo a la movilidad social: desafíos en torno al rol del profesor secundario en la implementación de la indagación científica como enfoque pedagógico, Estudios Pedagógicos XXXV, (1), 63-78, (2009)

Hake, R. Interactive-engagement versus traditional methods: A six-thousand-student survey of mechanics test data for introductory physics courses, American Journal of Physics, 66 (1), 64-74, (1998)

Hodson, D. Hacia un enfoque más crítico del trabajo de laboratorio, Enseñanza de las ciencias, 12(3), 299$313,(1994)$

Jaime, E. y Escudero, C., El trabajo experimental como posible generador de conocimiento en enseñanza de la física. Enseñanza de las ciencias: revista de investigación y experiencias didácticas, 29(3), 371-380, (2011)

Lazzari, M. Combinación de Aprendizaje Cooperativo e Individual en una Asignatura de Química de Materiales. Formación Universitaria, 7(4), 39-46, (2014)

López, A. y Tamayo, O., "Las prácticas de laboratorio en la enseñanza de las ciencias naturales". Revista Latinoamericana de Estudios Educativos, 1(8), 145-166, (2012)

Mordeglia, C. y Mengascini, A., Caracterización de prácticas experimentales en la escuela a partir del discurso de docentes de primaria y secundaria. Enseñanza de las ciencias: revista de investigación y experiencias didácticas, 32(2), 71-89, (2014)

Mosquera, A., La Física de los fluidos en el sistema circulatorio: Propuesta didáctica para comprender los fenómenos físicos de fluidos en el organismo humano. Monografía para optar el título de Magister en Enseñanza de las Ciencias Exactas y Naturales Universidad Nacional de Colombia. Bogotá (2011)

Parra, M. y Flores, R., Aprendizaje cooperativo en la solución de problemas con fracciones. Educación Matemática, 20(1), 31-52, (2008)

Szigety, E., Viau, J., Tintori, M. y Gibbs, H., Tensión superficial: un modelo experimental con materiales sencillos. Revista Eureka sobre Enseñanza y Divulgación de las Ciencias, 9 (3), 393-400, (2012)

Vera, F., Rivera, y Fuentes. R., La galería de Galileo: videos de experimentos para la enseñanza de la física. Revista Estudio Pedagógicos, 39, 143-151, (2013) 
